pass up because of the ileo-caecal valve, and not only will this cause pain and discomfort, but there is also considerable danger of the caecum bursting or perforating. This operation of short-circuiting an irremovable growth is well worth doing, for not only is malignant disease of the large bowel of slow growth, but when it is relieved from the irritation of faecal matter passing over it it will grow even more slowly. The way in which the growth destroys life early is, of course, by causing obstruction, and once this danger is removed by the short-circuit many patients live comfortably for a considerable time, often two or three year's; a colostomy, of course, achieves the same object, but it does so often with considerable permanent discomfort and distress to the patient.

Removable Growth Not Producing Obstruction.

We now come to our fourth and final class of case, in which there is no obstruction and the growth proves to be removable, and here our aim and object should be to excise the growth and reunite the ends of the bowel by some form of anastomosis straight away at one sitting. Even in this case, where the growth is removable, there are certain eminent authorities who prefer to make no attempt at all to remove the growth, but merely do the short-circuiting operation, described above, in all cases, for they say that the risk of performing a short-circuit is considerably less than that of removing the growth, and that if the growth is not removed, but a short-circuit is performed, the patient will probably live comfortably for two or three years; whereas even if the growth is removed he will probably suffer from a recurrence within two or three years. Theoretically these arguments are true enough, but personally I have no sympathy with the conclusions to which they lead, for by removing the growth we offer the patient a reasonable chance of a permanent cure, at a certain risk, and I think that most patients would prefer to face this rather than to have a certainty of death within two or three years and less immediate risk at the operation. Of course, if the patient is over 65 , or is feeble and not likely to live long in any case, we may then rightly choose the short-circuiting operation rather than the radical removal. The nature and scope of the operation and the.anastomosis which should be performed when removing a carcinoma of the colon depend naturally upon the situation and extent of the growth, and the same operation will be done whether it is being performed as a primary removal of a non-obstructed growth or as a secondary operation after acute obstruction has been relieved a week or two before. The procedure in general consists in mobilizing and freeing the growth and that part of the colon in which it lies, if necessary, and then removing the growth, a certain amount of bowel above and below it, and a deep V-shaped piece of the mesentery right down to the mesenteric root, and calculated to include all the glands and lymphatic vessels which drain that portion of the colon to be removed, all these structures being excised in one piece. As these growths appear to extend microscopically rather further up the bowel than down it; and to spread especially in the submucous layer, it is customary to remove rather more bowel above the growth than below it. The ends of the bowel are then joined together, either by lateral, end-to-end, or end-toside anastomosis, and it is a wise precaution to prevent an undue distension of the bowel above the junction with gas by making a temporary caecostomy as a safety valve. If the union is low down in the colon this object will be equally well achieved by stretching the anal sphincter, or by passing a flatus tube up from the anus through the anastomosis and leaving it in place for a few days.

So constant are these colonic growths in position that the operations which are performed for their removal are now more or less standardized. Thus for growths in the caecum or right-hand side of the colon it is necessary to remove the whole of both the caecum and the ascending colon in order to be certain of keeping outside the limits of the growth, and as by doing this we are liable to interfere with the blood supply of the lowest few inches of the ileum, and also to leave a blind pocket of transverse colon near the hepatic flexure, it has become customary in cases of carcinoma in this situation to remove the lowest five inches of the ileum, the caecum, the ascending colon, and a few inches of the transverse colon, and then to re-establish the continuity of the gut by uniting the ileum to the transverse colon by either side-to-side or end-to-end union. In this case there is no means of providing a safety valve above the anastomosis, nor is there any need so to do, as the faeces are here quite liquid. This operation must, of course, be preceded by mobilizing the caecum and ascending colon by means of an incision through the peritoneum of the posterior abdominal wall, on the outer side of the colon, and it will also entail a dissection of the lymphatics and glands up to the origin of the superior mesenteric artery. In the case of growths of the transverse and pelvic colons, where there is a well marked mesentery, simple resection of the growth with a deep $\mathrm{V}$ of the mesentery and end-toend union is sufficient; while if the carcinoma is in the descending colon or splenic flexure, as before, mobilization of the flexure and colon will be necessary, and then we must remove a few inches of the transverse colon, the flexure, and the whole of the descending colon. When dealing with portions of the large bowel which are not completely covered with peritoneum, such as the descending colon, it is well to remember that it is not wise to perform end-to-end anastomosis, and that lateral or end-to-side unions are preferable if possible. When performing a lateral or side-to-side anastomosis on the left of the colon; as after removal of the splenic flexure, I usually deliberately make an antiperistaltic anastomosis instead of the usual isoperistaltic one, for this reproduces to a certain extent the natural angle of the splenic flexure and turns the closed ends of the bowel and the raw edges where the mesentery has been cut, out into the retroperitoneal tissues and prevents them forming intraperitoneal adhesions.

Some knowledge of the relative degrees of malignancy of the three types of growth which are met with, and to which reference has been made above, is important; for in the most malignant type-the hard, craggy ulcer-one would feel justified in performing a more extensive and more radical operation than would be necessary for one of the other two less malignant varieties, and we can, during the operation, balance in our mind the extent of the procedure rendered necessary by the degree of malignancy of the particular type of growth present, and compare it with the patient's strength and ability to stand such a procedure.

The diagnosis of carcinoma of the colon in some ways resembles that of carcinoma of the stomach, for both are obvious in the later stages, but are difficult of diagnosis in the early stages, and this is the time when they should be subjected to operation if we are to offer the patient a reasonable chance of non-recurrence. Of both diseases the surgeon sees more cases which are inoperable than operable; this alone should be sufficient to make us feel that, on a reasonable suspicion that either of these diseases is present, an exploratory laparotomy is more than justifiable.

\section{THE CUTANEOUS TUBERCULIN TEST} applied to a Series of Definitely Diagnosed Caseg oF TUBERCULOSIS.

BY

S. L. CUMMINS, C.B., C.M.G., M.D.

DAVID DAVIES PROFESSOR OF TUBERCULOSIS, WELSH NATIONAL SCHOOR OF MEDICINE, CARDIFF, AND PRINCIPAL MEDICAL OFFICER, WELSH NATIONAL MEMORIAL ASSOCIATION.

In the British Medical Journal of April 21st, 1923 (p. 673), Dr. Charles McNeil describes a method of carrying out the cutaneous tuberculin test as follows:

"A small circular area of skin on the front of the forearm is chafed with the sharp point of a darning-needle until the epidermis is removed and the pink cutis vera exposed. Care is taken to avoid bleeding. The eye of the needle is now charged with undiluted old tuberculin, and pressed into the prepared vascular surface with a rotary motion. A small bruised pit is thus formed from which the tuberculin is rapidly absorbed."

In the course of an investigation into a series of cases of tuberculosis, some pulmonary, others surgical, under treatment in certain of the residential institutions of the Welsh 
National Memorial Association, it became necessary to ascertain the extent of their sensitivity to tuberculin, and the McNeil technique appeared to be a suitable one for the purpose. As, however, it was desired to obtain, at the hands of workers in three different institutions, results that should be comparable, it was clearly necessary to standardize the test as far as possible, especially as regarded the size of the area of scarification. A means of limiting the excursion of the needle-point to a definite area was found in the Abel Morrall metal gauge for knitting-needles, an instrument obtainable for a few pence, consisting of a plate of metal perforated with agraded series of apertures by which knittingneedles are measured. Aperture No. 3 was found to be of suitable dimensions for the test. For use, the metal gauge was pressed firmly against the surface of the forearm of the patient, so that the skin bulged up slightly into the circular aperture. Over the area of skin thus fixed and delimited the point of the needle was worked until a circular patch of cutis was laid bare. The gauge was now removed, leaving a scarified area of standard size into which the tuberculin was rubbed after the method of McNeil. The needles used were "Crescent" (sharp) carpet needles, which were chosen for their sharp points and capacious eves, suitable for carrying and "rubbing in" the charge of tuberculin.

In each case a series of six scarifications were made, three on each forearm, with a view to emploring five strengths of tuberculin and a control. The strengths of tuberculin used were as follows: 2, 4, 8, 16, and 32 per cent. These dilutions were prepared just before use by a "drop method" suggested by Professor Drerer, F.R.S., sterile normal saline solution being used as the diluent and also as the control fluid. This method is shown in the following table, published with the kind permission of Professor Dreyer.

Table I.-Professor Dreyer's Tuberculin Dilution Method.

\begin{tabular}{|c|c|c|c|c|c|}
\hline No. & Tukerculin. & & Saline. & Dilution. & $\begin{array}{l}\text { Strength (per } \\
\text { cent.) of } \\
\text { Tuberculin. }\end{array}$ \\
\hline 1 & 1 drop & + & $28 \mathrm{drops}$ & 1 in 43.4 & 2.02 \\
\hline 2 & 2 d:ops & + & 28 & 1 in 25.2 & 3.97 \\
\hline 3 & $4 \quad$ & + & 26 & 1 in 12.3 & 8.13 \\
\hline 4 & , & + & 12 & 1 in 6.2 & 16.1 \\
\hline 5 & .. & + & 10 & 1 in 3.2 & 31.5 \\
\hline
\end{tabular}

Saline to be dropped in first; dropping pipette cleaned and dried. Tuberculin dropped in. Saline and tuberculin carefully mixedwithout violent shaking. Same dropping pipette to be used for same series of dilutions.

(The dropping pipette used is that supplied for use in the Dreyer agglutination technique. The tuberculin dilutions should be made up freshly for each series of observations as alterations take place up reshly for each series of observations as alterations take place volumes of tuberculin and diluting fluid are drawn up into a syringe or pipette, successive diminutions of concentration being obtained by further dilutions measured with the same instrument there is a considerable fallacy, due to the retention of an indeterminat quantity of tuberculin on the inside of the syringe from the last concentration used. In Dreyer's method this concentration used. eliminated, a point of great importance where accurate comparisons of reactivity are sought.

The tuberculin selected for the tests was P.T.O. (B. W. and Co.). Phials of this preparation, together with standard dropping pipettes and sterile salt solution, Morrall gauges and carpet needles, were provided for each institution, together with exact details of the technique to be employed. The results of the tests were read after 24,48 , and 72 hours and on the fifth day.

In all, 80 cases were investigated; these included 24 advanced pulmonary and surgical cases tested by me at Glan Ely Hospital, near Cardiff ; 30 sanatorium cases, tested by Dr. H. E. Watson at the South Wales Sanatorium, Talgarth; and 26 patients, some pulmonary and some surgical, tested by Dr. D. A. Powell at the North Wales Sanatorium, Denbigh. The results are summarized in the following table.

It will be noticed that the severe cases, for the most part young phthisical women and "surgical" children, at Glan Ely Hospital and the North Wales Sanatorium, gare but a small percentage of "positives" with the more dilute tuber-

\begin{tabular}{c|c|c|c|c}
\multicolumn{5}{c}{ TABLE II. } \\
\hline $\begin{array}{c}\text { Tuberculin } \\
\text { Strength } \\
\text { (per cent.). }\end{array}$ & $\begin{array}{c}\text { Glan Ely } \\
\text { Hospital } \\
\text { (24 patients). } \\
\text { Positive. }\end{array}$ & $\begin{array}{c}\text { North Wales } \\
\text { Sanatoriun } \\
\text { (26 patients). } \\
\text { Positive. }\end{array}$ & $\begin{array}{c}\text { South Wales } \\
\text { Sanatorium } \\
\text { (30 patients). } \\
\text { Positive. }\end{array}$ & Total \\
(80 patients). \\
\hline 2 & 7 & 6 & 17 & $30=37.5 \%$ \\
4 & 6 & 7 & 21 & $34=42.5 \%$ \\
8 & 9 & 9 & 24 & $42=52.5 \%$ \\
16 & 24 & 20 & 27 & $71=88.7 \%$ \\
22 & 24 & 22 & 28 & $74=92.5 \%$
\end{tabular}

culin, though nearly all of them reacted to the concentrated doses. At Talgarth, however, where the patients were males with, for the most part, a more favourable type of disease, the proportion reacting with weak tuberculin was considerable.

The results of the tests were, on the whole, best seen after forty-eight hours, although, as in the cases reported by $\mathrm{McNeil}$, some of the reactions appeared late. In the majority of cases the reactions were fading or had faded by the fifth day, but some few persisted for several weeks. The relation of time and of the strength of tuberculin to the number of "positives" on each day of the observation is shown in Table IIT, based on the Talgarth cases only.

TABLE III. (Thirty cases.)

\begin{tabular}{c|c|c|c|c}
\hline $\begin{array}{c}\text { Tuberculin } \\
\text { Strength } \\
\text { (per cent.). }\end{array}$ & $\begin{array}{c}\text { After 24 } \\
\text { hours. }\end{array}$ & $\begin{array}{c}\text { After 48 } \\
\text { hours. }\end{array}$ & $\begin{array}{c}\text { After 72 } \\
\text { hours. }\end{array}$ & Fifth Day. \\
\hline 2 & 9 & 13 & 10 & 5 \\
4 & 13 & 18 & 15 & 8 \\
8 & 19 & 21 & 19 & 12 \\
16 & 23 & 27 & 22 & 15 \\
32 & 25 & 26 & $: 5$ & 19 \\
\hline
\end{tabular}

Here it is seen at once that the number of cases showing a positive reaction is highest in every tuberculin concentration after forty-eight hours; and that the numbers were already falling after seventy-two hours.

Amongst the patients investigated by me at Glan Ely Hospital the two cases that gave the feeblest reactions, both of them female patients with advanced pulmonary disease, died within three months of the tests. Another patient, a man suffering from advanced spinal disease as well as pulmonary tuberculosis, is now, at the end of three months from testing, rapidly nearing his end. The patient, a male pulmonary case, that gave the strongest reaction-c "double plus" with every dilution up to 2 per cent.- has greatly improved and is making rapid progress under treatment.

I learn from Dr. Powell that the patients found by him to give a negative or doubtful reaction were all severe cases, one with acute phthisis and two with advanced sacro-iliac disease complicated by sinus formation. These observations, taken with the tendency observed at Talgarth to well marked reactions even with the more dilute tuberculin, support the view, generally held, that a sharp reactivity is associated with good powers of resistance. At the same time it was observed that many severe and advanced cases reacted fairly well, especially to the stronger concentrations of tuberculin; and there is little doubt but that, had undiluted tuberculin been employed as recommended by McNeil, a positive response would have been evoked in every case. Out of the 80 patients tested, a positive response, weak or strong, was noted with at least one "strength" of tuberculin on one or more of the days of observation in all but three cases-that is to say, in 86.2 of the total.

My thanks are due to $\mathbf{D r}$. A. Brownlee, Medical Superintendent, Glan Ely Hospital, and to Dr. W. H. Tytler, Central Tuberculosis Laboratory, Cardiff, for their kind help, and to Dr. H. E. Watson of the South Wales Sanatorium and Dr. D. A. Powell of the North Wales Sanatorium for their co-operation in carrying out the tests and for their kindness in placing their results at my disposal. 